\title{
EFECTO DE UN SISTEMA DE PRODUCCIÓN QUÍMICA DE OXÍGENO EN CULTIVO DE BAGRE TIGRITO (Pimelodus pictus) EN SISTEMAS DE RECIRCULACIÓN SOMETIDOS A INTERRUPCIÓN DE LA AIREACIÓN MECÁNICA
}

Fecha de recepción: 12 de agosto de 2013 • Fecha de aceptación: 24 de octubre de 2013

\author{
EFFECT OF A CHEMICAL OXYGEN PRODUCTION SYSTEM IN CATFISH (Pimelodus \\ pictus) IN RECIRCULATION AQUACULTURE SYSTEMS SUBJECT TO INTERRUPTION \\ OF MECHANICAL AIREATION
}

Ana C. Torres-Mesa B.Sc ${ }^{11} \bullet$ Liliana Cifuentes-Torres B.Sc • Hernán Hurtado-Giraldo Ph.D1 • Edwin Gómez-Ramírez M.Sc ${ }^{1,2}$

\section{RESUMEN}

Los sistemas de cultivo intensivo de peces, son en términos generales dependientes de la energía eléctrica. Una interrupción del fluido eléctrico lleva rápidamente a la muerte de los organismos cultivados, por lo que se hace necesario contar con sistemas de emergencia, como las plantas eléctricas. Sin embargo una buena y funcional alternativa son los sistemas de producción química de oxígeno. En el presente trabajo se utilizó un sistema de producción de oxígeno gaseoso basado en la reacción entre el peróxido de hidrógeno y el agua. Para ello se montaron seis sistemas cerrados de recirculación de 250 l, compuestos por un tanque de peces, un clarificador y un biofiltro. En cada uno de los sistemas se mantuvieron 50 individuos de Pimelodus pictus. Los tratamientos T1 y T2, fueron sometidos a un corte de luz de 24 horas. Sin embargo al T1 se le incorporó un sistema de producción química de oxígeno, mientras que para T2 no hubo ningún tipo de aireación alterna. Cada uno de los tratamientos contaba con tres repeticiones. En todos los casos se midieron parámetros fisicoquímicos y se estimaron las variables de producción. En todos los sistemas los parámetros fisicoquímicos y de producción (exceptuando el oxígeno disuelto), se mantuvieron similares y dentro del rango aceptable para la especie. El oxígeno disuelto en el T1 mostró un nivel significativamente mayor que el de T2. Estos datos indican que el uso de sistemas de producción química de oxígeno pueden ser considerados como una alternativa de emergencia para ser empleados en sistemas cerrados de recirculación.

Palabras clave: demanda de oxígeno, peróxido de hidrógeno, catálisis.

Grupo de Ictiología, Laboratorio de Fisiología, Programa de Biología Aplicada, Facultad de Ciencias Básicas y Aplicadas, Campus Rio Grande, Cajicá, Cundinamarca, Colombia

2 Autor para correspondencia: edwin.gomez@unimilitar.edu.co 


\section{ABSTRACT}

Fish intensive culture systems are totally electricity dependent. An interruption in electricity results very fast in death of cultured organisms. In such emergency cases electrical generator are used, but an alternative is to use oxygen chemical production systems. In this work a gaseous oxygen chemical production system based on the reaction between hydrogen peroxide and water. In each of the systems, 50 Pimelodus pictus individuals were maintained. The T1 and T2 treatments were subjected to a power outage of 24 hours. However the T1 treatment had a chemical production system oxygen, whereas for T2 there was no alternative aeration. Each treatment had three replicates. In all systems physicochemical and production parameters were obtained. No differences in these parameters (excepted dissolved oxygen) were observed among treatments, and these values were within normal levels for the specie. For dissolved oxygen, T1 shows a significant higher level than T2. These data indicate that the oxygen chemical production systems can be used as alternative in recirculation systems.

Keywords: Oxygen demand, hydrogen peroxide, catalysis.

\section{INTRODUCCIÓN}

\section{Bagre tigrito}

El bagre tigrito ( $P$. Pictus) es una especie que cuenta con una amplia distribución, pudiéndose encontrar en Colombia en regiones como Amazonas, Meta, Arauca, Putumayo y Caquetá (Rubio, 2008). Es un pez considerado como estenotermo, prefiriendo temperaturas que oscilan entre los $25^{\circ}-28^{\circ} \mathrm{C}$ y pH entre 6.0 a 8.0 (Wisocky et al., 2006). Es una especie omnívora con tendencia a la carnivoría, de hábitos nocturnos que emplea sus barbillones para encontrar a sus presas. Respecto a su cultivo, se ha reportado que es relativamente fácil de mantener en sistemas de recirculación sencillos, presentando buenos niveles de crecimiento y supervivencia (Mondragón et al., 2012).

$P$. pictus es una especie que se comercializa como un pez ornamental, debido a su gran aceptación en el mercado internacional. Una evidencia de esto es que fue considerada como una de las principales

especies exportadas entre 1998 y 2004 (Blanco, 2002; Galvis et al., 2007).

\section{Sistemas de recirculación}

Los sistemas cerrados de recirculación (SCR) son una alternativa de producción acuícola en la que se usa de manera más racional el agua gracias a la incorporación de filtros mecánicos y biológicos que ayudan a disminuir la concentración de compuestos nitrogenados tóxicos y a reducir la cantidad de sólidos disueltos y suspendidos. Además, el bajo requerimiento de tierra e independencia del clima, la facilidad del tratamiento de algunas enfermedades y la reducción en la generación de desechos producidos por los peces, hacen de estos una tecnología más sustentable (Timmons y Ebeling, 2007). Sin embargo, una de las mayores dificultades que presentan los SCR es su total dependencia a la energía eléctrica (Masser et 
al., 1999) considerando que un corte de luz puede resultar en la muerte de la totalidad de los peces. Es por ello que se hace indispensable incorporar sistemas de energía de emergencia, como las plantas eléctricas.

La mortalidad de los peces ocasionada por un corte de energía se debe a que procesos claves para el mantenimiento de las condiciones fisicoquímicas del agua en el sistema, como los son la aireación y el movimiento del agua se realizan utilizando equipos eléctricos, cuyo funcionamiento permite que se cumplan funciones como la oxigenación, la remoción de partículas sólidas y la disminución en la concentración de nitrógeno amoniacal total (NAT) y nitrito $\left(\mathrm{NO}_{2}-\right)$ (Ebeling et al., 1995). Estos parámetros fisicoquímicos son vitales para mantener el buen estado de salud de los peces, su adecuado crecimiento y supervivencia (Gutierrez-Wing y Malone, 2006; Martins et al., 2009; Singer et al., 2008).

La aireación ayuda a mantener la concentración de oxígeno disuelto $(\mathrm{OD})$ requerida para el buen funcionamiento de peces y bacterias nitrificantes. El nivel de OD se considera uno de los factores limitantes en los sistemas de recirculación, por ello debe mantenerse al menos en 4-5 ppm. La remoción de sólidos en los sistemas de recirculación es fundamental para optimizar el funcionamiento de los biofiltros. No hacerlo, incrementa la demanda de oxígeno, reduciendo su concentración y disponibilidad en el sistema (McGee y Chichra, 2000; Brambilla et al., 2008). La remoción de amonio también depende de la concentración de $\mathrm{OD}$, ya que este proceso de filtración biológica es llevado a cabo por bacterias aeróbicas como los son $\mathrm{Ni}$ trosomonas y Nitrobacter, que transforman el amonio en nitrito y este en nitrato (Ling y Chen, 2005; Schreier et al., 2010). Una deficiente biofiltración, provocada por bajos niveles de OD puede conducir a la muerte de los peces, debido al exceso de amonio (especialmente de la forma no ionizada) y a la fijación de nitrito en las moléculas de hemoglobina inhibiendo el transporte de oxígeno en la sangre.

\section{Producción química de oxígeno}

Una posible alternativa como sistema de emergencia para la producción de oxígeno de manera química

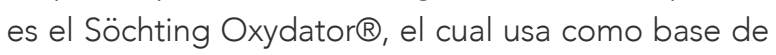
su funcionamiento el peróxido de hidrógeno $\left(\mathrm{H}_{2} \mathrm{O}_{2}\right)$ y catalizadores de cerámica (http://www.oxydator.de). En estudios previos se ha reportado que el $\mathrm{H}_{2} \mathrm{O}_{2}$ se puede emplear como desinfectante, sin dañar el medio ambiente y sin alterar la salud de los individuos presentes en los SCR (Pedersen y Pedersen, 2011). De igual manera, se ha evaluado su aplicación en biofiltros y se ha evidenciado que no influencia de manera negativa el proceso de nitrificación (Schwartz et al., 2000; Møller et al., 2010). Otros estudios reportan que puede ser usado como fuente de oxígeno para el transporte de peces, desde alevines hasta adultos, mostrando un incremento en la concentración de OD y una mayor sobrevivencia, comparativamente con el tratamiento control de cada uno de los estudios (Taylor y Ross, 1988; Barua et al., 2012).

Teniendo en cuenta que se ha reportado que puede llevarse a cabo la producción de oxígeno químico por medio de la interacción catalizador $-\mathrm{H}_{2} \mathrm{O}_{2}$ y la importancia de los niveles OD en los parámetros de calidad de agua de los SCR, se planteó como objeto del presente trabajo describir las curvas de degradación y recuperación de parámetros fisicoquímicos del agua en cultivos de $P$. pictus en sistemas de recirculación sometidos a interrupción de la aireación mecánica, con o sin sistemas de producción química de oxígeno gaseoso, para determinar qué tan viables pueden ser estos sistemas como alternativas de emergencia durante periodos de carencia de energía eléctrica.

\section{MATERIALES Y METODOS}

Este trabajo se realizó en el Laboratorio de Fisiología en la Estación Experimental Hacienda Río Grande de la Universidad Militar Nueva Granada sede Cajicá, ubicada a 2558 msnm y con una temperatura 
promedio anual de $12.9^{\circ} \mathrm{C}$ (Datos Estación Climática de la Universidad Militar Nueva Granada).

\section{Montaje y seguimiento de sistemas}

Para el montaje del SCR se utilizó un diseño adaptado de los sistemas propuestos por Ebeling et al, (1995), Johnson y Wardlow (1997), Leal et al, (2007) y Hernández et al, (2010). Los animales fueron adquiridos a proveedores localizados en Bogotá, Villavicencio y manejados de acuerdo con lo establecido por los cánones de la ética en la experimentación animal (STAL, 2012). A este respecto debe aclararse, que aunque $P$. pictus es una especie de otras cuencas, al ser mantenida en sistemas cerrados de recirculación de agua, no existe riesgo que los individuos alcancen cursos de agua naturales de la Sabana de Bogotá. Esto se garantiza por el hecho que el experimento se realiza en un laboratorio lejano de los cursos de agua, bajo techo, y en sistemas cerrados (Figura 1). En cada SCR se colocaron 50 ejemplares de P. pictus de 4-5 $\mathrm{cm}$ de longitud total, para un total de $187.43 \pm 0.99 \mathrm{~g}$ de biomasa. Los peces fueron alimentados al $6 \%$ de la biomasa (ajustado quincenalmente) con concentrado comercial al $45 \%$ de proteína bruta, y a una temperatura de $29^{\circ} \mathrm{C}$.

Se plantearon los siguientes grupos experimentales para llevar a cabo el estudio:

Tratamiento 1 (T1). Tres sistemas a los que se les realizó un corte en la aireación mecánica durante 24 horas, durante las cuales se mantuvieron con aireación química con dos equipos comerciales
Söchting Oxydator ${ }^{\circledR}$ modelo A, cada uno con dos catalizadores y $200 \mathrm{ml}$ de $\mathrm{H}_{2} \mathrm{O}_{2} 6 \%$, para producir oxígeno gaseoso.

Tratamiento 2 (T2). Tres sistemas control sufrieron los mismos cortes de luz que T1 pero no se incorporó aireación química ni mecánica.

Utilizando un espectrofotómetro Spectroquant ${ }^{\circledR}$ Multy, se tomaron datos de parámetros fisicoquímicos como $\mathrm{NAT}, \mathrm{NO}_{2}{ }^{-}, \mathrm{NO}_{3}{ }^{-}$, dureza general $(\mathrm{GH})$ y dureza de carbonatos $(\mathrm{KH})$ y con la ayuda de una sonda multiparamétrica Extech $®$ DO 700, se tomaron datos de $\mathrm{pH}$, conductividad, OD y temperatura, cada 30 minutos, durante las tres primeras horas. Posteriormente, se midieron los parámetros cada hora hasta que se cumplieron 8 horas del corte y finalmente, una vez pasadas las 24 horas establecidas para la experimentación se introdujo nuevamente la aireación mecánica a los sistemas y se midieron los mismos parámetros. A los 30 días, se llevaron a cabo mediciones para calcular algunos parámetros productivos (Tabla 1) con el fin de establecer cuál fue la afectación a nivel productivo del corte de aireación efectuado.

\section{Sistema de producción química de oxígeno}

La base de este sistema de producción de oxígeno es el uso de $\mathrm{H}_{2} \mathrm{O}_{2}$, y su reacción con el agua en presencia de un catalizador, para producir oxígeno gaseoso. Se utilizó un equipo comercial Söchting xydator ${ }^{\circledR}$ tipo $A$, con dos catalizadores para cada sistema, ya que según las especificaciones técnicas

Tabla 1. Parámetros productivos calculados en este estudio.

\begin{tabular}{ccc}
\hline Parámetro & SIGLAS & Fórmula \\
\hline Ganancia de peso & GP & $\mathrm{p}_{\mathrm{f}}-\mathrm{p}_{\mathrm{i}}$ \\
\hline Tasa de crecimiento especifico & TCE & 100* $\left[\left(\operatorname{Ln} \mathrm{p}_{\mathrm{f}}-\operatorname{Ln~} \mathrm{p}_{\mathrm{i}}\right) / \mathrm{t}\right]$ \\
\hline Factor de conversión alimenticia & FCA & Alimento consumido / incremento peso \\
\hline
\end{tabular}

$p_{f}=$ peso final; $p_{i}=$ peso inicial; $t=$ tiempol; $L n=$ logaritmo natural (Mercado et al, 2006; Pineda, 1999; Salazar y Ocampo, 2002) 


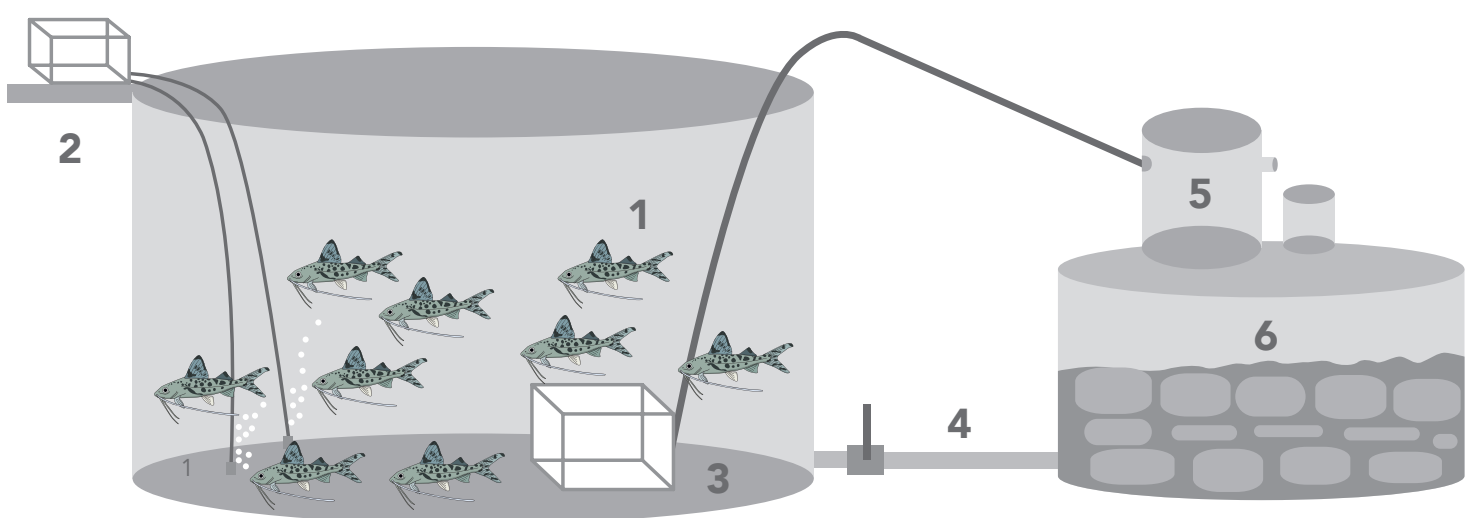

Figura 1. Sistema Cerrado de Recirculación. 1. Tanque de cultivo. 2 Aireador. 3. Bomba de agua sumergible. 4. Tubería. 5. Clarificador y filtro mecánico. 6. Biofiltro.

del equipo este tamaño es el adecuado para los sistemas de 250 litros. Este equipo permite una liberación controlada de oxígeno gaseoso, que difunde en todo el sistema. En presencia de los catalizadores (material cerámico mezclado con plata en polvo) una solución de $\mathrm{H}_{2} \mathrm{O}_{2}$ se convierte en $\mathrm{O}_{2}$ y $\mathrm{H}_{2} \mathrm{O}$, siguiendo la siguiente reacción:

\section{$2 \mathrm{H}_{2} \mathrm{O}_{2} \longrightarrow \mathrm{O}_{2}+2 \mathrm{H}_{2} \mathrm{O}$}

(http://www.aquariumoxygenator.com/atent\%20 4,784,765\%20Aquarium_oxygenator.pdf). Cada uno de los equipos empleados puede generar unos 540 $\mathrm{mg} \mathrm{O}_{2}$ diariamente.

\section{Análisis estadístico}

Se realizó un diseño experimental completamente aleatorizado (DCA) balanceado, con efecto interactivo del tiempo. Se realizó una prueba de Shapiro-Wilk para validar los supuestos de normalidad. Para determinar diferencias en las variables de crecimiento (peso, longitud total y estándar) se realizó una prueba de análisis de varianza y una prueba de comparación de medias (Tukey-Kramer) entre los tratamientos evaluados (Zar, 1999). Los parámetros fisicoquímicos, productivos y tasa de excreción de NAT fueron analizados con la prueba de $F$, para evaluar la homogeneidad de varianzas $y$, seguidamente una prueba de t. Se consideró una probabilidad del $5 \%(P<0.05)$ como diferencia significativa entre los tratamientos. Para el análisis se utilizó el program R de libre disposición en internet (http://www.r-project.org/)

\section{RESULTADOS Y DISCUSIÓN}

Al inicio del estudio los valores de OD inmediatamente antes de los cortes de energía eléctrica fueron de $6.40 \pm 017 \mathrm{mg} / \mathrm{l}$ para $\mathrm{T} 1$, y de $6.30 \pm 0.20 \mathrm{mg} / \mathrm{l}$ para T2 sin encontrar diferencias significativas entre ellos $(p>0,05)$. Al realizar el corte de aireación mecánica, la concentración de OD en los SCR para T1 durante los primeros $30 \mathrm{~min}$, se redujo a $5.53 \pm 0.25$ $\mathrm{mg} / \mathrm{l}$ y a $4.76 \pm 0.15 \mathrm{mg} / \mathrm{l}$ para $\mathrm{T} 2$, siendo significativamente más bajo el valor en T2 ( $<0.05)$. Estos valores se mantuvieron constantes durante las siguientes 24 h (Fig. 2: A). Con respecto a T1, el comportamiento del OD, fue contrario a lo reportado previamente 
por Pedersen y Pedersen (2012), donde la concentración de $\mathrm{OD}$ aumenta al aplicar $\mathrm{H}_{2} \mathrm{O}_{2}$ directamente en el biofiltro del sistema. Este sistema de aplicación del peróxido es diferente al utilizado en el presente trabajo, en el cual la generación de $\mathrm{O}_{2}$, se hace a través del sistema de catálisis del Oxydator $\Theta_{\text {, que }}$ bajo las condiciones utilizadas solo logró mantener

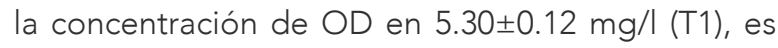
decir con pequeña disminución con respecto a nivel original. Esto puede deberse a que el sistema de catálisis (Oxydator $\left.{ }^{\circledR}\right)$ permite que el tiempo de generación de $\mathrm{O}_{2}$ sea mayor, ya que su reacción es controlada y por lo tanto la concentración de $\mathrm{O}_{2}$ en el agua se mantiene constante y por mayor tiempo, mientras que la aplicación directa de peróxido utilizada por Pedersen y Pedersen (2012), libera $\mathrm{O}_{2}$ de manera casi inmediata duplicando la concentración de este durante un periodo aproximado de dos horas solamente. Este incremento en el nivel de $\mathrm{O}_{2}$ en el agua puede variar ya que se ha demostrado que existe una relación directamente proporcional entre la concentración del $\mathrm{H}_{2} \mathrm{O}_{2}$ y la reacción de este compuesto con el agua (Wong, et al, 2010). Los valores de OD tanto T1 como T2 se encontraban dentro del rango mínimo aceptable para el manejo de $\mathrm{SCR}$, sin embargo el nivel de OD logrado y mantenido con la producción química de este gas se mantuvo más alta (T1).

Desafortunadamente no se cuenta con curvas de cinética de producción de $\mathrm{O}_{2}$ por estos equipos, pero teniendo en cuenta que se utilizaron dos catalizadores, y $200 \mathrm{ml}$ de peróxido $6 \%$ por oxydator, en estas condiciones se produce un máximo aproximado de 1080 mg O $/$ /día (http://www.oxydator.es/files/ oxydator_a.pdf). Es decir que se aportan aproximadamente 0,18 mg por litro en cada hora, apoyando la hipótesis de un aporte lento y bajo a los sistemas. No se tienen datos sobre los requerimientos de $\mathrm{O}_{2}$ de $P$. pictus y no se puede estimar que tan importante es el aporte dado por el peróxido. Pero si se considera lo que se esperaría como nivel de $\mathrm{O}_{2}$ por simple difusión a las condiciones de temperatura del agua y presión atmosférica utilizadas, que el OD se encontrara a un nivel aproximado de $5,5 \mathrm{mg} / \mathrm{l}$ o un poco menos en ausencia de peces (Yanez, 1976), esto indica que la producción química de $\mathrm{O}_{2}$ esta añadiendo al sistema suficiente oxígeno para mantener los peces, y evitar la baja que se observa en T2.

Por otro lado, los valores de $\mathrm{NAT}, \mathrm{NO}_{2}-$ y $\mathrm{NO}_{3}-$ (Fig. 2: $B, C$ y D) no mostraron diferencias significativas $(p>0.05)$ entre T1 y T2, manteniendo las concentraciones dentro de los rangos adecuados de cultivo (Timmons y Ebeling, 2007). El NAT para ambos tratamientos se vio incrementado por el corte de aireación mecánica durante las primeras $4 \mathrm{~h}$. Esto indica una disminución del funcionamiento de las poblaciones bacterianas nitrificantes. Aunque se ha reportado que la adición de $\mathrm{H}_{2} \mathrm{O}_{2}$ perjudica el adecuado funcionamiento de dichas poblaciones (Avendaño-Herrera et al, 2006; Pedersen et al, 2006), este no parece ser el caso en este trabajo, ya que la disminución en los sistemas sin peróxido es similar. Adicionalmente en esos estudios el $\mathrm{H}_{2} \mathrm{O}_{2}$ se adicionaba directamente al sistema, mientras que para el presente experimento se llevó a cabo la incorporación de oxígeno químico de manera indirecta, a través de un sistema de catálisis.

Las concentraciones de $\mathrm{NO}_{2}$ - tendieron a disminuir para luego estabilizarse. Una posible causa de la disminución inicial, es la interacción entre este anión y el $\mathrm{O}_{2}$ (el cual proviene de la catálisis de $\mathrm{H}_{2} \mathrm{O}_{2}$ ) el cual pueden llegar a producir peroxinitrito (ONOO-) (Beckman y Koppenol, 1996; Jiang y Yuan, 2013). Esto pudo sumarse con la segunda etapa de la nitrificación, es decir la transformación del $\mathrm{NO}_{2}{ }^{-}$en $\mathrm{NO}_{3}{ }^{-}$, para disminuir aún más el nivel de $\mathrm{NO}_{2}-$. Esto es consistente con la presencia de $\mathrm{NO}_{3}$ - en los sistemas. Se observó que tanto para $\mathrm{NO}_{2}$ - como para $\mathrm{NO}_{3}$-, la concentración mantenida durante todo el tiempo de experimentación estaba 
Cinética de Oxígeno

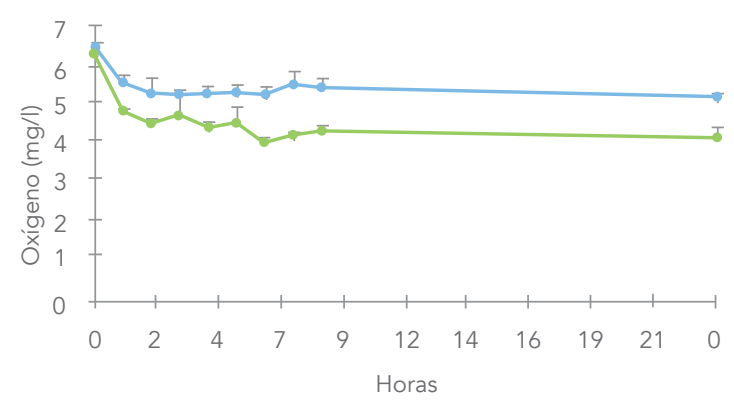

Cinética del $\mathrm{NO}_{2}$ -

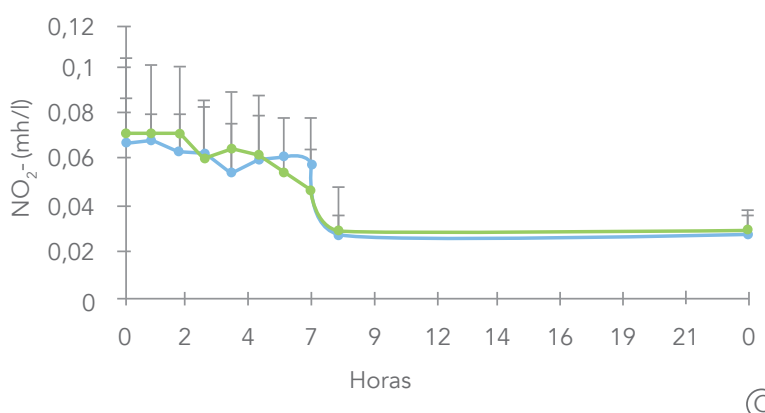

Cinética del NAT

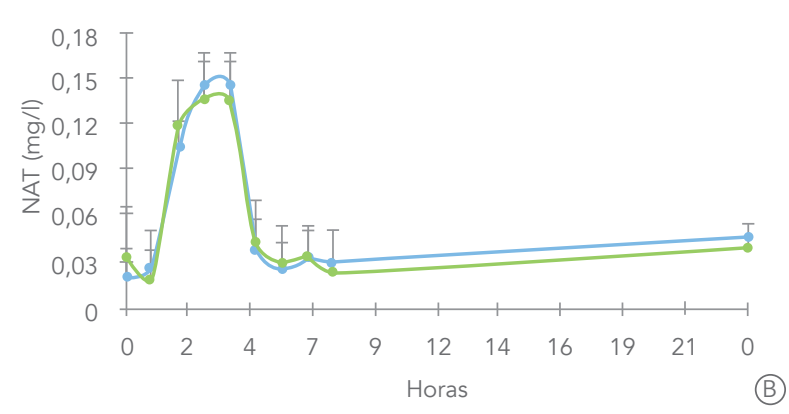

Dinámica del $\mathrm{NO}_{3}-$

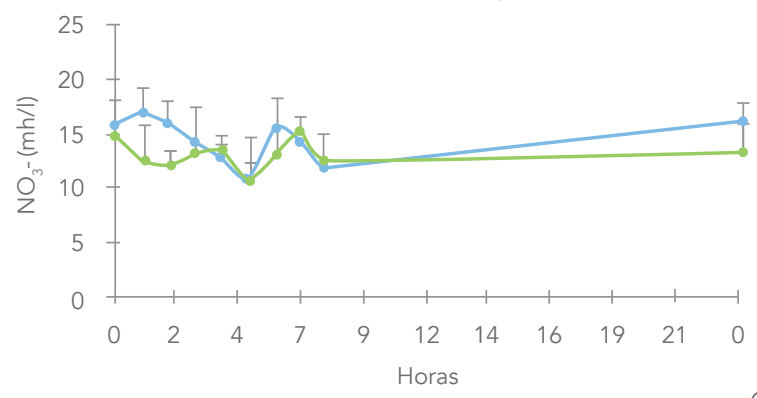

Figura 2. Comportamiento de las concentraciones de OD, NAT, $\mathrm{NO}_{2}$ - y NO $\mathrm{NO}_{3}$ - en $\mathrm{SCR}$ de $\mathrm{T} 1$ (Azul) y $\mathrm{T} 2$ (Verde). Cada valor representa el promedio, \pm la desviación estándar.

dentro del rango considerado inofensivo para los peces (Timmons y Ebeling, 2007).

Una pregunta que surge a partir de este trabajo es por qué no se presentaron diferencias en los valores de la mayoría de los parámetros fisicoquímicos, independientemente del tratamiento. Es evidente que los niveles de OD, que son indispensables para mantener muchos de esos parámetros, fueron adecuados, como lo muestran los datos, tanto con aireación química como sin ella. Para el caso de los sistemas con $\mathrm{H}_{2} \mathrm{O}_{2}$, existen dos fuentes para el OD. La primera, es la conversión de peróxido $\mathrm{a}_{2}$, mientras que la segunda corresponde a la difusión de $\mathrm{O}_{2}$ atmosférico. En los sistemas sin peróxido, solo se contaría con esta última. De estos resultados se desprende que, bajo las condiciones utilizadas, y teniendo en cuenta que la biomasa contenida por los sistemas es bastante baja, la demanda de $\mathrm{O}_{2}$ también será baja, lo cual explicaría que pueda ser suplida por simple difusión. Pero por otro lado si es claro que se presenta un nivel de OD mayor en los sistemas con producción química incorporada. Esto también indica que para biomasas mayores, la presencia extra de $\mathrm{O}_{2}$ generado por el peróxido podría ser importante.

En cuanto a otros parámetros fisicoquímicos (temperatura, $\mathrm{pH}, \mathrm{KH}$ y $\mathrm{GH}$ ), estos se midieron al principio del experimento y se observó que permanecieron dentro de los límites tolerables para la especies de acuerdo con lo reportado por Mondragón et al (2012), por lo que se conservaron para ambos tratamientos sin presentar diferencias significativas ( $p>0.05)$, para así mantener las condiciones estables.

Los parámetros de crecimiento (peso, longitud estándar y total) y de productividad (Ganancia 
Tabla 2. Valor promedio de temperatura, $\mathrm{pH}$, dureza general $(\mathrm{GH})$ y dureza de carbonatos $(\mathrm{KH})$. Cada valor representa el promedio, \pm la desviación estándar.

\begin{tabular}{ccc}
\hline & T1 & T2 \\
\hline $\begin{array}{c}\text { Temperatura } \\
\left({ }^{\circ} \mathrm{C}\right)\end{array}$ & $29.22 \pm 0.39$ & $29.74 \pm 0.67$ \\
\hline $\mathrm{pH}$ & $6.47 \pm 0.13$ & $6.67 \pm 0.31$ \\
\hline $\mathrm{GH}(\mathrm{mg} / \mathrm{l})$ & $179 \pm 18.44$ & $187 \pm 25.07$ \\
\hline $\mathrm{KH}(\mathrm{mg} / \mathrm{l})$ & $17.9 \pm 0$ & $17.9 \pm 0$ \\
\hline
\end{tabular}

en peso, Tasa de crecimiento específico, Factor de conversión alimenticia y sobrevivencia) (Tabla 3), no mostraron diferencias significativas entre los tratamientos ( $p>0.05$ ). Sin embargo se evidencia un menor crecimiento respecto con el trabajo de Mondragón et al (2012), probablemente debido a la diferencia de tamaño de los individuos para los dos estudios, ya que la tasas de crecimiento son mayores en individuos más pequeños, por ser metabólicamente más activos (Ip et al., 2001; Freedman y Noakes, 2002). Al comparar los resultados obtenidos en este trabajo con los de Baquero (2008), en donde se cultivó $P$. pictus en estanques de tierra por 100 días, y Obando (2009) quien levantó los individuos en acuarios de 50 I durante un periodo de 45 días, se observa que se obtuvieron valores de crecimiento y productividad menores a los reportados en el presente estudio, estas diferencias en el crecimiento de los individuos posiblemente fueron causadas por las condiciones de mantenimiento de los individuos, ya que específicamente en el trabajo de Obando (2009) mencionan que los individuos se mantuvieron bajo continuos recambios de agua en los acuarios debido a que los fisicoquímicos no eran los adecuados para el mantenimiento de $P$. pictus.

Sin embargo, las necesidades básicas de los individuos parece que se mantuvieron de manera adecuada, ya que se logró un crecimiento relativamente bueno y una buena supervivencia comparativamente con trabajos anteriores (Baquero, 2008; Obando, 2009; Mondragón, et al, 2012).

La utilización de procedimientos de generación química de oxígeno permite mantener un nivel de OD mayor que el observado sin aireación mecánica o química. Esto coincide con lo reportado por Hossain et al (2012), quienes demostraron la utilidad del $\mathrm{H}_{2} \mathrm{O}_{2}$ como sistema de oxigenación en el transporte de alevinos de tilapia nilótica. El uso de $\mathrm{H}_{2} \mathrm{O}_{2}$ en cultivos de camarón en sistema biofloc, también apoya

Tabla 3. Parámetros de crecimiento y productividad. Cada valor representa el promedio, \pm la desviación estándar.

\begin{tabular}{cccccc} 
& T1 & T2 & $\begin{array}{c}\text { Mondragón } \\
\text { et al, 2012 }\end{array}$ & $\begin{array}{c}\text { Baquero, } \\
\mathbf{2 0 0 8}\end{array}$ & $\begin{array}{c}\text { Obando, } \\
\mathbf{2 0 0 9}\end{array}$ \\
\hline Peso inicial (g) & $5.84 \pm 0.65$ & $5.27 \pm 0.62$ & $1.18 \pm 0.32$ & 0.8 & $2.17 \pm 0.75$ \\
\hline Longitud inicial (cm) & $7.89 \pm 0.70$ & $7.75 \pm 0.62$ & $4.97 \pm 0.51$ & 3.6 & $6.74 \pm 0.78$ \\
\hline Peso final (g) & $6.58 \pm 1.79$ & $5.57 \pm 1.62$ & $6.23 \pm 0.77$ & 1.9 & $3.2 \pm 0.31$ \\
\hline Longitud final (cm) & $9.30 \pm 0.86$ & $9.00 \pm 0.58$ & $10.72 \pm 0.7$ & & $7.24 \pm 0.23$ \\
\hline GP (g) & $3.67 \pm 0.47$ & $3.61 \pm 0.39$ & 11.01 & 0.6 & \\
\hline TCE & $4.17 \pm 0.44$ & $4.22 \pm 0.27$ & & & \\
\hline FCA & $1.15 \pm 0.15$ & $1.13 \pm 0.12$ & 2 & $97.8-98.6$ \\
\hline S (\%) & 100 & 100 & $96-98$ &
\end{tabular}


el beneficio de la aplicación de este compuesto a bajas concentraciones como generador de oxígeno (Furtado et al, en prensa). Aunque no existen lineamiento de medida sobre la cantidad de $\mathrm{H}_{2} \mathrm{O}_{2}$ liberado por estos sistemas, la acción del proceso químico parece ser lenta, dado que no puede evitar la generación de un pico inicial de NAT. Esta baja liberación también explicaría que no se afecta significativamente las bacterias responsables de la nitrificación en los sistemas, y se mantienen bajos los niveles de NAT y $\mathrm{NO}_{2}$-. Esta es una preocupación, debido a su muy conocido efecto bactericida (Yanong, 2011). Sin embargo, estudios indican que este riesgo es muy bajo y que la degradación del $\mathrm{H}_{2} \mathrm{O}_{2}$ es rápida en presencia de materia orgánica, como es el caso en sistemas de recirculación. Este último punto es importante para otra inquietud que puede surgir al respecto del uso del peróxido en acuicultura, a saber su efecto sobre el ambiente y sobre los humanos que consuman estos productos. El hecho que la FDA ya autorizó la utilización de este compuesto en actividades de acuicultura (FDA, 2007) apunta a que ya se reconoce que bien manejado es inocuo tanto para el ambiente como para los consumidores humanos.

\section{AGRADECIMIENTOS}

A la Vicerrectoría de Investigaciones de la Universidad Militar Nueva Granada, proyecto CIAS-936.

\section{BIBLIOGRAFÍA}

23. Avendaño-Herrera, R, Magariños, $B$, Irgang, R y Toranzo, AE. 2006. Use of hydrogen peroxide against the fish pathogen Tenacibaculum maritimum and its effect on infected turbot (Scophthalmus maximus). Aquaculture, 257: 104-110.

24. Aya E, Arias C. 2011. Reproducción inducida de Pimelodus pictus con extracto de hipófisis de carpa (EHC) y Ovaprim $®$. Revista Medicina Veterinaria y Zootécnica Córdoba. 16:2317-2323.

25. Baquero H. 2008. Respuesta del Tigrito Pimelodus pictus a dos sistemas de contención y de alimentación en la etapa de levante. Trabajo de grado. Facultad de Zootecnia. Universidad de la Salle. Bogotá - Colombia, pg 24-68.

26. Beckman, JS y Koppenol, WH. 1996. Nitric oxide, superoxide, and peroxynitrite: the good, the bad, and ugly. American Journal of Physiology, 271: C1424-1437.

27. Barua P, Awal A, Zamal H. 2012. Effective Role of Hydrogen Peroxide as an Oxygen Source for Fingerlings of Oreochromis niloticus During Transportation in Bangladesh. Journal of Life Sciences. 6 (1): 1 - 56.

28. Blanco C. 2002. Consideraciones sobre los peces ornamentales de Colombia. En: Mojica-Corso JI, C. Castellanos-Castillo JS. Usma-Oviedo y R. Álvarez- León (eds.). El libro rojo de los peces dulceacuícolas de Colombia. La Serie de Libros Rojos de Especies Amenazadas de Colombia. ICNUNC / IIBAVH / MINAMBIENTE / Cl-Colombia. Bogotá. Colombia; p. 47-54. 
29. Brambilla F, Antonini M, Ceccuzzi P, Terova G, Saroglia M. 2008. Foam fractionation efficiency in particulate matter and heterotrophic bacteria renoval from a recirculating seabass (Dicentracchus labrax) system. Aquacultural. Engineering. 39:37-42.

30. Ebeling J, Jensen G, Losordo T, Masser M, McMullen J, Pfeiffer L, Rakocy J, Sette M. 1995. Model Aquaculture recirculation system (MARS). Engineering and operations manual. Department of Agricultural Education and Studies. lowa State University. USA.

31. FDA. 2007. FDA removes hydrogen peroxide from the list of low regulatory priority aquaculture drugs. http://www.fda.gov/AnimalVeterinary/ Consultado el 7 de enero de 2014.

32. Freedman JA, Noakes DLG. 2002. Why are there no really big bony fishes? a point-of-view on maximun body size in teleosts and elasmobranchs. Kluwer Academic Publishers, 12: 403 - 416.

33. Furtado PS, Serra FP, Poersch LH, Wasielesky W. (en prensa). Acute toxicity of hydrogen peroxide in juvenile white shrimp Litopenaeus vannamei reared in biofloc technology systems. Aquaculture International.

34. Galvis V, Mojica C, Provenzano R, Lassoalcalá C, Taphorn D, Royero R, Castellanos C. 2007. Peces de la Orinoquia colombiana con énfasis en especies de interés ornamental. Instituto Colombiano de Desarrollo Rural INCODER y Universidad Nacional de Colombia. Primera edición.

35. Gutierrez-Wing MT, Malone RF. 2006. Biological filters in aquaculture : trends and research directions for freshwater and marine applications. Aquacultural Engineering. 34 :163-171.
36. Hernandez C, Ramírez E, Hurtado H. 2010. Estudio preliminar del levante de juveniles de arawana plateada Osteoglossum bicirhossum en sistemas cerrados de recirculación. Revista Facultad de Ciencias Univeridad Militar Nueva Granada. 6:96-113.

37. Hossain Z, Abdul A, Prabal B. 2012. Effective role of hydrogen peroxide as an oxygen source for fingerlings of Oreochromis niloticus during transportation in Bangladesh. Journal of Life Sciences, 6:56.

38. Imues MA, Sánchez D. 2013. Selección de hembras reproductoras de pez tigrito (Pimelodus pictus), utilizando análisis discriminantes. Revista de Investigación Pecuaria, 2:29-37.

39. Ip YK, Chew SF y Randall DJ. 2001. Ammonia toxicity, tolerant and excretion. En: Wright $\mathrm{P}$, Anderson P (Editores). Nitrogen excretion. Academia Press, EEUU, p. 109 - 148.

40. Jiang, G y Yuan, Z. 2013. Synergistic inactivation of anaerobic wastewater biofilm by free nitrous acid and hydrogen peroxide. Journal of Hazardous Materials, 250-251: 91-98.

41. Johnson DM, Wardlow GW. 1997. A prototype Recirculating aquaculture-hydroponic system. University of Arkansas. Department of Agricultural \& Extension education.

42. Leal A, Sanchez VC, Rodríguez D, Moreno P, Gómez E, Hurtado H. 2007. Estudio preliminar del efecto de la temperatura del agua sobre el crecimiento de Carassius auratus mantenido en sistemas de recirculación. Revista de la Facultad de Ciencias Universidad Militar Nueva Granada. 3:163-175. 
43. Ling J, Chen S. 2005. Impact of organic carbon on nitrification performance of different biofilters. 2005. Aquacultural Engineering. 33:150-162.

44. Martins CIM, Ochola D, Ende SSW, Eding EH, Verreth JAJ. 2009. Is growth retardation present in Nile tilapia Oreochromis niloticus cultured in low water exchange recirculating aquaculture systems?. Aquaculture. 298:43-50.

45. Masser MP, Rakocy J, Losordo TM. 1999. Recirculating aquaculture tank production systems. Management of recirculating systems. SRAC Publication 452. Southern Regional Aquaculture Center, USA.

46. McGee M, Cichra, C. 2000. Principles of water recirculation and filtration in aquaculture. Document FA12. Institute of Food and Agricultural Sciences. Extension. University of Florida, USA.

47. Mercado Bl; García JL; Rosado RC; Olaya CN; Segura FG; Brú SC and Tordecilla GP. Cultivo de Dorada (Brycon sinuensis Dahl, 1995) en Jaulas Flotantes a Diferentes Niveles de Proteína. Revista Colombiana de Ciencias Pecuarias. 2006; 19: 204 - 211.

48. Mondragón AM, Torres A, Cifuentes L, Becerra D, Hurtado H, Gómez E. 2012. Evaluación de sistema cerrado de recirculación para el levante de bagre tigrito (Pimelodus pictus). Revista de la Facultad de Ciencias Básicas Universidad Militar Nueva Granada, 8:96-107.

49. Olaya CM, Ovalle CH, Gómez E, Rodríguez D, Caldas ML, Hurtado H. 2007. Histología y morfometría de sistema digestivo del Silúrido bagre tigrito (Pimelodus pictus). Revista de Medicina Veterinaria y Zootecnia. 2007. 54:311-323.
50. Obando A. 2009. Evaluación de cuatro densidades de acopio sobre la sobrevivencia y el desarrollo productivo del tigrito (Pimelodus pictus) en la Estación Piscícola la Terraza, ViIlavicencio, Meta. Trabajo de Grado. Programa de Zootecnia. Facultad de Ciencias Agropecuarias. Universidad de la Salle. Bogotá-Colombia. 14-59.

51. Pedersen, L-F, Pedersen, PB y Sortkjær, O. 2006. Dose-dependent decomposition rate constants of hydrogen peroxide in small-scale bio filters. Aquacultural Engineering, 34: 8-15.

52. Pedersen LF, Pedersen LB. 2012. Hydrogen peroxide application to a comercial recirculating aquaculture system. 2012. Aquacultural Engineering, 46:40-46.

53. Pineda R. Elaboración Y Evaluación De Dietas A Partir De Harinas De Barrilete (Euthynnus linneatus) Y Rasposa (Haemulon maculiconda) Como Alimento De Bagre (Ictalurus punctatus) En Condiciones De Laboratorio. Tesis de Maestría. Universidad Colima - Mexico. 1999. p 20-22.

54. Rahman MM; Nagelkerke LAJ; Verdegem MCJ; Wahab MA and Verrth JAJ. Relationship among Water Quality, Food Resources, Fish Diet and Fih Growth in Polycultured Ponds: A Multivariate Approach. Aquaculture. 2008; 275:108-115.

55. Remen M; Imsland AK; Stefansson SO; Jonassen TM and Foss A. Interactive Effects of Ammonia and Oxygen on Growth and Physiology Status of Juvenile Atlantic cod (Gadus morhua). Aquaculture. 2008; 274: 292 - 299.

56. Rubio EA. 2008. Introducción a los peces dulceacuícolas de Colombia. Facultad de 
Ciencias Exactas y Naturales, Universidad del Valle, Cali, Colombia.

57. Salazar MB and Ocampo D. Tasa De Crecimiento Del Pez Ángel Pterophyllum scalre (Perciformes: Chiclidae) En Condiciones De Laboratorio. Acta Universitaria. 2002; 12: 28-33.

58. Schreier HJ, Mirzoyan N, Wsaito K. 2010. Microbial Diversity of biological filter inrecirculating aquaculture Systems. Current Opinions in Biotechnology. 21:1-8.

59. Schwartz MF, Bullock GL, Hankins JA, Summerfelt ST, Mathias JA. 2000. Effects of selected chemo- therapeutants on nitrification in fluidized-sand biofilters for coldwater fish production. International Journal of Recirculating Aquaculture. 1: 61-81.

60. Singer A, Parnes S, Gross A, Sagi A, Brenner A. 2008. A novel approach to denitrification processes in a zero-dischargerecirculating system for small scale urban aquaculture. Aquacultural Engineering. 39:72-77.

61. STAL.2012. Harmonisation internationale des lignes directrices concernant la revue éthique des propositions relatives à l'utilization des animaux, l'enseignement et la formation des utilizateurs d'animaux por la science. 38, 2ème trimester.

62. Timmons MB, Ebeling JM. 2007. Recirculating Aquaculture. Cayuga Aqua Ventures. New York, USA.

63. Turnbull RD, Timmons MB. 1993. Use of biological filters in recirculating aquaculture systems. Extension Bulletin 463. Department of Agriculture and Biological Engineering. Cornell University, Ithaca, NY.
64. Wisocky L, Montey K, Popper A. 2006. The influence of ambient temperature and thermal acclimation on hearing in a eurythermal and a stenothermal otophysan fish. Journal of Experimental Biology, 212:3091-3099.

65. Wong, GTF, Wu, Y-C y Li, K-Y. 2010. Winkler's method overestimates dissolved oxygen in natural waters: Hydrogen peroxide interference and its implications. Marine Chemistry, 122: 83-90.

66. Yanes F. Diseño de plantas de tratamiento de aguas residuales para países en desarrollo. Lima, CEPIS, 1976m pg 1-65. (http://www.bvsde.paho.org/bvsacd/scan2/05862/05682-11. pdf). Consultado el 8 de enero de 2014.

67. Yanong RP.I 2011. Use of hydrogen peroxide in finfish aquaculture. FA157. University of Florida. IFAS extension

68. Zar JH. Biostatistical analysis. Fourth Edition, Prentice Hall, 1999. 